\title{
能動膝関節と半円足を持つリムレスホイールの平地動歩行
}

\section{Level Dynamic Walking of Rimless Wheel with Active Knees and Semicircular Feet}

\author{
浅野 文彦 （北陸先端科学技術大学院大学） \\ $\bigcirc$ 大島 正高 （北陸先端科学技術大学院大学）
}

Fumihiko ASANO, Japan Advanced Institute of Science and Technology, fasano@jaist.ac.jp Masataka OHSHIMA, Japan Advanced Institute of Science and Technology, ohshima_09@jaist.ac.jp

\begin{abstract}
The authors clarified that the measurable period of double-limb support (DLS) motion emerges in passive dynamic walking of a viscoelastic-legged rimless wheel (RW) through experiments and numerical simulations. This paper then defines the conditions for transition to DLS motion and discusses the potentiality for the emergence of DLS in limit cycle walking with knees. We numerically show that the measurable period of DLS emerges during the phase after impact where the knee joints are free.
\end{abstract}

Key Words: Limit cycle walking, Rimless wheel, Knee joint, Double-limb support

\section{1. はじめに}

McGeer の受動歩行 [1][2] をはじめとして, リミットサイク ル型歩行ロボットの衝突モデルは「後脚は前脚の接地と同時に 速やかに床面から離れる」という仮定の下で導かれるのが一般 的であった.この非弹性衝突モデルの妥当性は, コンパス型歩 行器とこれに膝関節を付加したものについて, 1990 年の段階 で McGeerにより理論と実験の両面から確認されている。 ま た, 後続研究においても理論的に再考されることは無く, これ まで有効なモデルとして用いられてきた. 結果として, リミッ トサイクル型歩容の連続時間運動は常に単脚支持状態（Singlelimb support ; 以下 SLS）であり，これに瞬間的な両脚支持状態 （Double-limb support；以下 DLS）が加わることで成立する周 期運動であると広く認識されるようになった。

これに対して筆者らは, 粘弾性脚を持つ Rimless wheel（以下 RW）の受動歩行において衝突直後から DLS が発現することを 数値シミュレーションと実験の両面から確認した [3]. 解析を通 して, 脚フレームが直動関節を持つ場合には, McGeer のモデル は妥当でないという一つの具体例を示した. しかしながら, その 数学モデルは実験で勧測された「前脚が床面に衝突しても後脚 が離陸しない」という事実を直感的に適用したレベルのもので あり, 脚の粘性係数が大きい場合には正しく機能しない可能性 も示唆された。

以上を踏まえ本稿では，以下の二つの問題を考察する.

(1) 運動が DLS/SLS に移行する数学的条件の定式化

（2）直動脚でない（膝関節を持つ）歩行モデルにおけるDLS の 発現可能性

問題 (2) を考察するために, 能動膝関節を持つ RW のモデルを 解析の対象として導入する. その衝突方程式の力学的意味を再 考し, 未定乗数べクトルの各成分の符号を確認することで, 前脚 の接地から DLS/SLS への移行の判定が行えることを説明する. また，導かれた判定条件に従うことで，DLSを含む平地歩行の 数值シミュレーションが可能となることを示す.
2. モデリング

\section{$2 \cdot 1$ 仕様}

本稿では図 1 に示す歩行モデルを扱う，以下の仮定をおく.

・回転関節を持つ 8 本の脚リンクから構成される対称形状で ある。

・滕関節より下 (下腿) のリンクの質量を $m[\mathrm{~kg}]$ とする. 半 円足の質量もこれに含まれる.

・本体の質量を $m_{H}[\mathrm{~kg}]$ とする. 重心回りの慣性モーメント は考慮しない。

- 隣り合う脚フレーム間の相対角度を $\alpha=\pi / 4[\mathrm{rad}]$ とする.

- 両脚支持状態における前脚を Leg 1 , 後脚を Leg 2 と呼ぶ.

- Leg 1 の先端位置（半円足の取り付け位置）を $(x, z)$ とする.

- Leg 1 の絶対角度を $\theta_{1}$, 膝関節の相対角度を $\theta_{2}, \operatorname{Leg} 2$ の 膝関節の相対角度を $\theta_{3}$ とする. 時計回りを正回転とする.

- Leg $i$ の膝関節には回転駆動力 $u_{i}$ を印加できるものとする. 他の 6 脚の滕関節は角度 $\beta[\mathrm{rad}]$ に固定され, 運動しないも のとする.

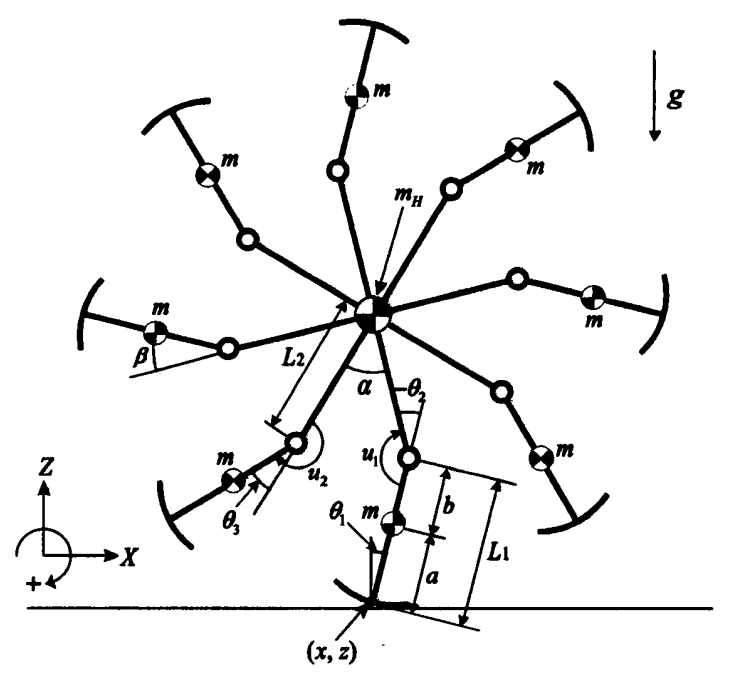

Fig. 1 Model of planar rimless wheel with active knees and semicircular feet 


\section{$2 \cdot 2$ 運動方程式}

一般化座標ベクトルを $\boldsymbol{q}^{\mathrm{T}}=\left[\begin{array}{lllll}x & z & \theta_{1} & \theta_{2} & \theta_{3}\end{array}\right]$ とする と, ロボットの運動方程式は

$$
\begin{aligned}
\boldsymbol{M}(\boldsymbol{q}) \ddot{\boldsymbol{q}}+\boldsymbol{h}(\boldsymbol{q}, \dot{\boldsymbol{q}}) & =\boldsymbol{S} \boldsymbol{u}+\boldsymbol{J}(\boldsymbol{q})^{\mathrm{T}} \boldsymbol{\lambda} \\
\boldsymbol{J}(\boldsymbol{q}) \dot{\boldsymbol{q}} & =\mathbf{0}
\end{aligned}
$$

で与えられる. 式 (1) の右辺第一項は膝関節トルクベクトルで あり

$$
\boldsymbol{S u}=\left[\begin{array}{ll}
0 & 0 \\
0 & 0 \\
0 & 0 \\
1 & 0 \\
0 & 1
\end{array}\right]\left[\begin{array}{l}
u_{1} \\
u_{2}
\end{array}\right]
$$

である．また，右辺第二項は床面と足裏の間に作用するホロノ ミック拘束力項であり, 式 (3) はその速度拘束条件式である. 式 (1)(2) より未定乗数べクトル $\boldsymbol{\lambda}$ を消去すると, 次のように整理 される.

$$
\begin{aligned}
\boldsymbol{M}(\boldsymbol{q}) \ddot{\boldsymbol{q}}= & \boldsymbol{Y}(\boldsymbol{q})(\boldsymbol{S} \boldsymbol{u}-\boldsymbol{h}(\boldsymbol{q}, \dot{\boldsymbol{q}})) \\
& -\boldsymbol{J}(\boldsymbol{q})^{\mathrm{T}} \boldsymbol{X}(\boldsymbol{q})^{-1} \dot{\boldsymbol{J}}(\boldsymbol{q}, \dot{\boldsymbol{q}}) \dot{\boldsymbol{q}} \\
\boldsymbol{Y}(\boldsymbol{q}):= & \boldsymbol{I}_{5}-\boldsymbol{J}(\boldsymbol{q})^{\mathrm{T}} \boldsymbol{X}(\boldsymbol{q})^{-1} \boldsymbol{J}(\boldsymbol{q}) \boldsymbol{M}(\boldsymbol{q})^{-1}
\end{aligned}
$$

\section{$2 \cdot 3$ 拘束条件の遷移}

結果を先に述べれば，生成される DLS を含む歩行運動は次の 三つの相から構成される.

- 衝突相 (Leg 1 と床面との衝突)

- 両脚支持期 (1 自由度)

- 単脚支持期 (3 自由度)

各相において，式 (1) のヤコビアン $\boldsymbol{J}(\boldsymbol{q})$ を以下に述べるそれに 切り換える.

\subsection{1 両脚支持期}

$$
\text { 次の二条件 : }
$$

(C1) Leg 1 の足裏が床面に対して滑らず転がりながら接して いる

(C2) Leg 2 の足裏が床面に対して滑らず転がりながら接して いる

から四つの速度拘束条件式が導かれ，これらをまとめるとヤコ ビアンが

$$
\begin{aligned}
& \boldsymbol{J}_{\mathrm{DLS}}(\boldsymbol{q})=\left[\begin{array}{ccccc}
1 & 0 & R \cos \theta_{1}-R & 0 & 0 \\
0 & 1 & -R \sin \theta_{1} & 0 & 0 \\
1 & 0 & J_{33} & J_{34} & J_{35} \\
0 & 1 & J_{43} & J_{44} & J_{45}
\end{array}\right] \\
& J_{33}=L_{1} \cos \theta_{1}+L_{2} \cos \left(\theta_{1}+\theta_{2}\right)-L_{2} \cos \left(\theta_{1}+\theta_{2}+\alpha\right) \\
& -\left(L_{1}-R\right) \cos \left(\theta_{1}+\theta_{2}+\theta_{3}+\alpha\right)-R \\
& J_{34}=L_{2} \cos \left(\theta_{1}+\theta_{2}\right)-L_{2} \cos \left(\theta_{1}+\theta_{2}+\alpha\right) \\
& -\left(L_{1}-R\right) \cos \left(\theta_{1}+\theta_{2}+\theta_{3}+\alpha\right)-R \\
& J_{35}=-\left(L_{1}-R\right) \cos \left(\theta_{1}+\theta_{2}+\theta_{3}+\alpha\right)-R \\
& J_{43}=-L_{1} \sin \theta_{1}-L_{2} \sin \left(\theta_{1}+\theta_{2}\right)+L_{2} \sin \left(\theta_{1}+\theta_{2}+\alpha\right) \\
& +\left(L_{1}-R\right) \sin \left(\theta_{1}+\theta_{2}+\theta_{3}+\alpha\right) \\
& J_{44}=L_{2} \sin \left(\theta_{1}+\theta_{2}\right)+L_{2} \sin \left(\theta_{1}+\theta_{2}+\alpha\right) \\
& +\left(L_{1}-R\right) \sin \left(\theta_{1}+\theta_{2}+\theta_{3}+\alpha\right) \\
& J_{45}=\left(L_{1}-R\right) \sin \left(\theta_{1}+\theta_{2}+\theta_{3}+\alpha\right)
\end{aligned}
$$

と求まる.この相では， 5 自由度のシステムに 4 つの拘束が加 わっているため, 実質的に 1 自由度の運動となる. 式 (1)のヤコ ビアンを各行ごとに

$$
\boldsymbol{J}_{\mathrm{DLS}}(\boldsymbol{q})=\left[\begin{array}{c}
\boldsymbol{J}_{1}(\boldsymbol{q}) \\
\boldsymbol{J}_{2}(\boldsymbol{q}) \\
\boldsymbol{J}_{3}(\boldsymbol{q}) \\
\boldsymbol{J}_{4}(\boldsymbol{q})
\end{array}\right]
$$

と定義するとき，ホロノミック拘束力は

$$
\begin{aligned}
\boldsymbol{J}_{\mathrm{DLS}}(\boldsymbol{q})^{\mathrm{T}} \boldsymbol{\lambda}= & \boldsymbol{J}_{1}(\boldsymbol{q})^{\mathrm{T}} \lambda_{1}+\boldsymbol{J}_{2}(\boldsymbol{q})^{\mathrm{T}} \lambda_{2}+\boldsymbol{J}_{3}(\boldsymbol{q})^{\mathrm{T}} \lambda_{3} \\
& +\boldsymbol{J}_{4}(\boldsymbol{q})^{\mathrm{T}} \lambda_{4}
\end{aligned}
$$

と分解される. $\boldsymbol{J}_{1}, \boldsymbol{J}_{2}$ は $(\mathrm{C} 1)$ の拘束条件に, $\boldsymbol{J}_{3}, \boldsymbol{J}_{4}$ は $(\mathrm{C} 2)$ のそれに対応するものである. Leg 1 の脚先に作用する拘束力の 鉛直方向成分 $\lambda_{2}$ は床反力に等しく, Leg 2 のそれは $\lambda_{4}$ となる. Leg 2 が床面から浮上する瞬間は， $\lambda_{4}$ の符号を観測することで 検知することができる. $\lambda_{2} \geq 0$ かつ $\lambda_{4} \geq 0$ であることは DLS の必要条件である.

\section{3 .2 単脚支持期}

ここでは条件 $(\mathrm{C} 1)$ のみが作用するため, ヤコビアンは

$$
\boldsymbol{J}_{\mathrm{SLS}}(\boldsymbol{q})=\left[\begin{array}{l}
\boldsymbol{J}_{1}(\boldsymbol{q}) \\
\boldsymbol{J}_{2}(\boldsymbol{q})
\end{array}\right]
$$

となる.

\section{4 衝突方程式}

非弾性衝突モデルを解く前に衝突直前の状態量 $\boldsymbol{q}^{-}, \dot{\boldsymbol{q}}^{-}$を, Leg 1 と Leg 2 を次のそれへと置き換えた $\boldsymbol{q}^{\dagger}, \dot{\boldsymbol{q}}^{\dagger}$ に修正する必 要がある. 具体的には以下のように定まる.

$$
\begin{aligned}
\boldsymbol{q}^{\dagger}= & {\left[\begin{array}{c}
x^{\dagger} \\
z^{\dagger} \\
\theta_{1}^{-}+\theta_{2}^{-}-\alpha+\beta \\
-\beta \\
-\theta_{2}^{-}
\end{array}\right], \dot{\boldsymbol{q}}^{\dagger}=\left[\begin{array}{c}
\dot{x}^{\dagger} \\
\dot{z}^{\dagger} \\
\dot{\theta}_{1}^{-}+\dot{\theta}_{2}^{-} \\
0 \\
-\dot{\theta}_{2}^{-}
\end{array}\right] } \\
x^{\dagger}= & x^{-}+L_{1} \sin \theta_{1}^{-}+L_{2} \sin \left(\theta_{1}^{-}+\theta_{2}^{-}\right) \\
& -L_{2} \sin \left(\theta_{1}^{-}+\theta_{2}^{-}-\alpha\right)-L_{1} \sin \left(\theta_{1}^{-}+\theta_{2}^{-}-\alpha+\beta\right) \\
z^{\dagger}= & z^{-}+L_{1} \cos \theta_{1}^{-}+L_{2} \cos \left(\theta_{1}^{-}+\theta_{2}^{-}\right) \\
& -L_{2} \cos \left(\theta_{1}^{-}+\theta_{2}^{-}-\alpha\right)-L_{1} \cos \left(\theta_{1}^{-}+\theta_{2}^{-}-\alpha+\beta\right) \\
\dot{x}^{\dagger}= & \dot{x}^{-}+L_{1} \cos \theta_{1}^{-} \dot{\theta}_{1}^{-}+L_{2} \cos \left(\theta_{1}^{-}+\theta_{2}^{-}\right)\left(\dot{\theta}_{1}^{-}+\dot{\theta}_{2}^{-}\right) \\
& -L_{2} \cos \left(\theta_{1}^{-}+\theta_{2}^{-}-\alpha\right)\left(\dot{\theta}_{1}^{-}+\dot{\theta}_{2}^{-}\right) \\
& -L_{1} \cos \left(\theta_{1}^{-}+\theta_{2}^{-}-\alpha+\beta\right)\left(\dot{\theta}_{1}^{-}+\dot{\theta}_{2}^{-}\right) \\
\dot{z}^{\dagger}= & \dot{z}^{-}-L_{1} \sin \theta_{1}^{-} \dot{\theta}_{1}^{-}-L_{2} \sin \left(\theta_{1}^{-}+\theta_{2}^{-}\right)\left(\dot{\theta}_{1}^{-}+\dot{\theta}_{2}^{-}\right) \\
& +L_{2} \sin \left(\theta_{1}^{-}+\theta_{2}^{-}-\alpha\right)\left(\dot{\theta}_{1}^{-}+\dot{\theta}_{2}^{-}\right) \\
& +L_{1} \sin \left(\theta_{1}^{-}+\theta_{2}^{-}-\alpha+\beta\right)\left(\dot{\theta}_{1}^{-}+\dot{\theta}_{2}^{-}\right)
\end{aligned}
$$

これより衝突直前の状態量は $\boldsymbol{q}^{\dagger}, \dot{\boldsymbol{q}}^{\dagger}$, 衝突直後のそれは $\boldsymbol{q}^{+}, \dot{\boldsymbol{q}}^{+}$ となる。

前脚が床面に衝突する際に両脚先が床面から受ける力を $\boldsymbol{f}$ と すると，運動方程式は

$$
M(q) \ddot{q}+h(q, \dot{q})=S u+J_{I}(q)^{\mathrm{T}} f
$$


となる. 時刻 $t=T_{0}[\mathrm{~s}]$ で衝突が起きたとすると, 式 (11) の左 辺の時間積分は

$$
\lim _{\varepsilon \rightarrow+0} \int_{T_{0}-\frac{\varepsilon}{2}}^{T_{0}+\frac{\varepsilon}{2}}(\boldsymbol{M}(\boldsymbol{q}) \ddot{\boldsymbol{q}}+\boldsymbol{h}(\boldsymbol{q}, \dot{\boldsymbol{q}})) \mathrm{d} t=\boldsymbol{M}\left(\boldsymbol{q}^{\dagger}\right)\left(\dot{\boldsymbol{q}}^{+}-\dot{\boldsymbol{q}}^{\dagger}\right)
$$

となる. 右辺の時間積分は, 制御入力が有界であることを考慮す ると

$$
\begin{aligned}
& \lim _{\varepsilon \rightarrow+0} \int_{T_{0}-\frac{\varepsilon}{2}}^{T_{0}+\frac{\varepsilon}{2}}\left(\boldsymbol{S u}+\boldsymbol{J}_{I}(\boldsymbol{q})^{\mathrm{T}} \boldsymbol{f}\right) \mathrm{d} t=\lim _{\varepsilon \rightarrow+0} \int_{T_{0}-\frac{\varepsilon}{2}}^{T_{0}+\frac{\varepsilon}{2}} \boldsymbol{J}_{I}(\boldsymbol{q})^{\mathrm{T}} \boldsymbol{f} \mathrm{d} t \\
& =\boldsymbol{J}_{I}\left(\boldsymbol{q}^{\dagger}\right)^{\mathrm{T}} \lim _{\varepsilon \rightarrow+0} \int_{T_{0}-\frac{\varepsilon}{2}}^{T_{0}+\frac{\varepsilon}{2}} \boldsymbol{f} \mathrm{d} t=\boldsymbol{J}_{I}\left(\boldsymbol{q}^{\dagger}\right)^{\mathrm{T}} \boldsymbol{\lambda}_{I}
\end{aligned}
$$

となる.以上より衝突方程式は

$$
\begin{aligned}
& \boldsymbol{M}\left(\boldsymbol{q}^{\dagger}\right) \dot{\boldsymbol{q}}^{+}=\boldsymbol{M}\left(\boldsymbol{q}^{\dagger}\right) \dot{\boldsymbol{q}}^{\dagger}+\boldsymbol{J}_{I}\left(\boldsymbol{q}^{\dagger}\right)^{\mathrm{T}} \boldsymbol{\lambda}_{I} \\
& \boldsymbol{J}_{I}\left(\boldsymbol{q}^{\dagger}\right) \dot{\boldsymbol{q}}^{+}=\mathbf{0}
\end{aligned}
$$

とまとめられ，これを解くことで衝突直後の速度べクトルが

$$
\begin{aligned}
& \dot{\boldsymbol{q}}^{+}=\left(\boldsymbol{I}_{5}-\boldsymbol{M}\left(\boldsymbol{q}^{\dagger}\right)^{-1} \boldsymbol{J}_{I}\left(\boldsymbol{q}^{\dagger}\right)^{\mathrm{T}} \boldsymbol{X}_{I}\left(\boldsymbol{q}^{\dagger}\right)^{-1} \boldsymbol{J}_{I}\left(\boldsymbol{q}^{\dagger}\right)\right) \dot{\boldsymbol{q}}^{\dagger} \\
& \boldsymbol{X}_{I}\left(\boldsymbol{q}^{\dagger}\right):=\boldsymbol{J}_{I}\left(\boldsymbol{q}^{\dagger}\right) \boldsymbol{M}\left(\boldsymbol{q}^{\dagger}\right)^{-1} \boldsymbol{J}_{I}\left(\boldsymbol{q}^{\dagger}\right)^{\mathrm{T}}
\end{aligned}
$$

と求まる. $J_{I}(\boldsymbol{q})$ は以下の手順に従って選択される.

(A1) 衝突方程式 (11) に扔ける $J_{I}(q)$ を $J_{\mathrm{DLS}}(\boldsymbol{q})$ として $\lambda_{I} \in$ $\mathbb{R}^{4}$ を計算する。

(A2) $\lambda_{I}$ を $\lambda_{I}=\left[\begin{array}{llll}\lambda_{I 1} & \lambda_{I 2} & \lambda_{I 3} & \lambda_{I 4}\end{array}\right]^{\mathrm{T}}$ と分解すると, DLS に移行するためには $\lambda_{I 2}>0$ かつ $\lambda_{I 4}>0$ であ ることが必要である.ここで $\lambda_{I 2}>0$ は自明であるので, 実際には $\lambda_{I 4}$ の符号のみを確認すれば良い.

(A3) $\lambda_{I 4}<0$ であれば DLS に移行しないので, $J_{I}(q)$ を $\boldsymbol{J}_{\mathrm{SLS}}(\boldsymbol{q})$ として $\dot{q}^{+}$を計算する.

(A4) $\lambda_{I 4} \geq 0$ であれば DLS に移行するので, $\boldsymbol{J}_{I}(\boldsymbol{q})$ を $\boldsymbol{J}_{\mathrm{DLS}}(\boldsymbol{q})$ として $\dot{\boldsymbol{q}}^{+}$を計算する.

\section{$2 \cdot 5$ 後脚の離陸の判定条件}

後脚の離陸は, $\lambda_{4}$ が連続的に正から負へ切り替わる瞬間に開 始する. 問題は, 膝関節トルクを印可し続けながら衝突する場合 には, 衝突時に $\lambda_{I 4}$ が正となっても衝突直後に $\lambda_{4}^{+}$が引き続き正 にならないことである.このことを考慮して, 本稿では以下の手 順に従った計算を行う。

(B1) $\lambda_{I 4} \geq 0$ で DLS に移行すると判断した場合, 衝突直後の 連立運動方程式は

$$
\begin{aligned}
\boldsymbol{M}\left(\boldsymbol{q}^{\dagger}\right) \ddot{\boldsymbol{q}}^{+}+\boldsymbol{h}\left(\boldsymbol{q}^{\dagger}, \dot{\boldsymbol{q}}^{+}\right) & =\boldsymbol{S} \boldsymbol{u}+\boldsymbol{J}_{\mathrm{DLS}}\left(\boldsymbol{q}^{\dagger}\right)^{\mathrm{T}} \boldsymbol{\lambda}^{+} \\
\boldsymbol{J}_{\mathrm{DLS}}\left(\boldsymbol{q}^{\dagger}\right) \dot{\boldsymbol{q}}^{+} & =\mathbf{0}_{4 \times 1}
\end{aligned}
$$

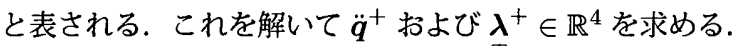

(B2) $\lambda^{+}$を $\lambda^{+}=\left[\begin{array}{llll}\lambda_{1}^{+} & \lambda_{2}^{+} & \lambda_{3}^{+} & \lambda_{4}^{+}\end{array}\right]^{\mathrm{T}}$ と分解するとき, $\lambda_{2}^{+} \geq 0$ かつ $\lambda_{4}^{+} \geq 0$ であれば (B1) で求めた $\ddot{q}^{+}$を用いて 引き続き数值積分を行う.

(B3) $\lambda_{2}^{+} \geq 0$ かつ $\lambda_{4}^{+}<0$ であれば一方向拘束が成立していな いため, (B1) で求めた $\ddot{\boldsymbol{q}}^{+}$を破棄し, 次の連立運動方程式

$$
\begin{aligned}
\boldsymbol{M}\left(\boldsymbol{q}^{\dagger}\right) \ddot{\boldsymbol{q}}^{+}+\boldsymbol{h}\left(\boldsymbol{q}^{\dagger}, \dot{\boldsymbol{q}}^{+}\right) & =\boldsymbol{S} \boldsymbol{u}+\boldsymbol{J}_{\mathrm{SLS}}\left(\boldsymbol{q}^{\dagger}\right)^{\mathrm{T}} \boldsymbol{\lambda}^{+} \\
\boldsymbol{J}_{\mathrm{SLS}}\left(\boldsymbol{q}^{\dagger}\right) \dot{\boldsymbol{q}}^{+} & =\mathbf{0}_{2 \times \mathbf{1}}
\end{aligned}
$$

を用いて $\ddot{q}^{+}$を再計算し，これを衝突直後の初期加速度と して数值積分を開始する.

\section{3. 制御系設計}

運動が DLSに移行したと判定された場合，以下のような切り 替え制御を行うことで歩容生成を行う.

\section{$3 \cdot 1$ 制御相 I}

衝突時刻を $t=0[\mathrm{~s}]$ とする.このとき, 膝関節トルクは印 可せず自由関節の状態とする．このまま自由運動を行うことで DLS が継続する.

\section{$3 \cdot 2$ 制御相 II}

時刻 $t=T_{1}(>0)[\mathrm{s}]$ に Leg 2 を離陸させるためのトルクを両 膝に印可し始める. 具体的には, Leg 2 の下腿が大きく跳ね上が るよう, 次のトルク

$$
\boldsymbol{u}=\left[\begin{array}{l}
u_{1} \\
u_{2}
\end{array}\right]
$$

を印可する. ただし， $u_{1}>0$ かつ $u_{2}>0$ である.

\section{3 制御相 III}

時刻 $t=T_{2}\left(>T_{1}\right)[\mathrm{s}]$ より, 以下に述べる両膝の出力 $\mathrm{PD}$ 制 御を開始する.これにより次の衝突姿勢へと整定される.

以下に出力追值制御系を設計する. 出力ベクトルを

$$
\boldsymbol{y}:=\left[\begin{array}{c}
\theta_{2} \\
\theta_{3}
\end{array}\right]=\boldsymbol{S}^{\mathrm{T}} \boldsymbol{q}
$$

とすると, その 2 階微分は

$$
\begin{aligned}
\ddot{\boldsymbol{y}}= & \boldsymbol{S}^{\mathrm{T}} \ddot{\boldsymbol{q}} \\
= & \boldsymbol{S}^{\mathrm{T}} \boldsymbol{M}(\boldsymbol{q})^{-1} \boldsymbol{Y}(\boldsymbol{q})(\boldsymbol{S} \boldsymbol{u}-\boldsymbol{h}(\boldsymbol{q}, \dot{\boldsymbol{q}})) \\
& -\boldsymbol{S}^{\mathrm{T}} \boldsymbol{M}(\boldsymbol{q})^{-1} \boldsymbol{J}(\boldsymbol{q})^{\mathrm{T}} \boldsymbol{X}(\boldsymbol{q})^{-1} \dot{\boldsymbol{J}}(\boldsymbol{q}, \dot{\boldsymbol{q}}) \dot{\boldsymbol{q}} \\
= & \boldsymbol{A}(\boldsymbol{q}) \boldsymbol{u}-\boldsymbol{B}(\boldsymbol{q}, \dot{\boldsymbol{q}})
\end{aligned}
$$

となる.ただし，

$$
\begin{aligned}
\boldsymbol{A}(\boldsymbol{q}):= & \boldsymbol{S}^{\mathrm{T}} \boldsymbol{M}(\boldsymbol{q})^{-1} \boldsymbol{Y}(\boldsymbol{q}) \boldsymbol{S} \\
\boldsymbol{B}(\boldsymbol{q}, \dot{\boldsymbol{q}}):= & \boldsymbol{S}^{\mathrm{T}} \boldsymbol{M}(\boldsymbol{q})^{-1}(\boldsymbol{Y}(\boldsymbol{q}) \boldsymbol{h}(\boldsymbol{q}, \dot{\boldsymbol{q}}) \\
& \left.+\boldsymbol{J}(\boldsymbol{q})^{\mathrm{T}} \boldsymbol{X}(\boldsymbol{q})^{-1} \dot{\boldsymbol{J}}(\boldsymbol{q}, \dot{\boldsymbol{q}}) \dot{\boldsymbol{q}}\right)
\end{aligned}
$$

である. $\ddot{y}=v$ を達成する制御入力は

$$
\boldsymbol{u}=\boldsymbol{A}(\boldsymbol{q})^{-1}(\boldsymbol{v}+\boldsymbol{B}(\boldsymbol{q}, \dot{q}))
$$

で定まり, $v$ を

$$
\boldsymbol{v}=-\boldsymbol{K}_{D} \dot{\boldsymbol{y}}+\boldsymbol{K}_{P}\left(\boldsymbol{y}_{\mathrm{d}}-\boldsymbol{y}\right)
$$

とすれば $\boldsymbol{y} \rightarrow \boldsymbol{y}_{\mathrm{d}}$ が実現される. ただし,

$$
\boldsymbol{y}_{\mathrm{d}}=\left[\begin{array}{c}
\theta_{2 \mathrm{~d}} \\
\theta_{3 \mathrm{~d}}
\end{array}\right]=\left[\begin{array}{c}
-\beta \\
\beta
\end{array}\right]
$$

である. なお，この相においては，次の衝突までに $\theta_{2}, \theta_{3}$ がそ れぞれの目標值 $\theta_{2 \mathrm{~d}}, \theta_{3 \mathrm{~d}}$ へと十分に収束するよう, PD ゲイン を大きく設定する. 


\section{4. 数值シミュレーション}

図 2 に平地動歩行のシミュレーション結果を示す.ロボットの 物理パラメータは表 1 のように設定した．また，衝突直後に両膝 が自由関節になるよう, 各制御相の切り替え時刻を $T_{1}=0.08$, $T_{2}=0.13[\mathrm{~s}]$ と設定た. この結果, $\lambda_{I 4}, \lambda_{2}^{+}, \lambda_{4}^{+}$の全てが正值 となり，衝突直後から DLS に移行すると判定された，図 3 は 3 歩分のスティツク線図（ただし Leg1，2のみ表示）である. 制御 相 II における膝関節トルクは $u_{1}=20.0, u_{2}=0.10[\mathrm{~N} \cdot \mathrm{m}]$ と した. 制御相 III においては，目標姿勢に十分な精度で整定して 倒れ込むことができるよう，PD ゲイン行列を次のように設定 した.

$$
\boldsymbol{K}_{D}=\left[\begin{array}{cc}
60 & 0 \\
0 & 60
\end{array}\right], \quad \boldsymbol{K}_{P}=\left[\begin{array}{cc}
900 & 0 \\
0 & 900
\end{array}\right]
$$

目標とする衝突姿勢は, 両膝を真直ぐに伸ばした状態 $(\beta=0$ [rad]) とした.

図 2(a) より，両膝が衝突直後から順方向に曲がり始めている ことが分かる. (c) より, 衝突直後から僅かな期間（制御相 I）に

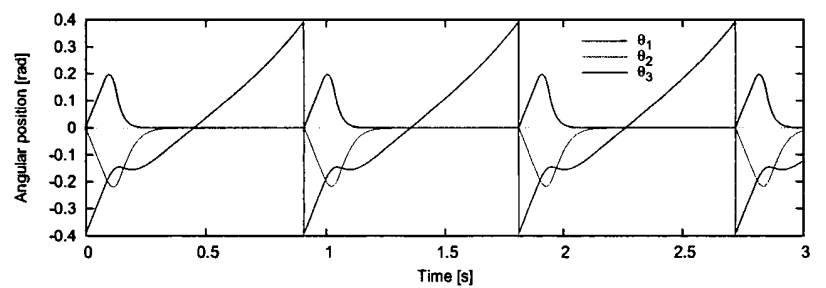

(a) Angular position

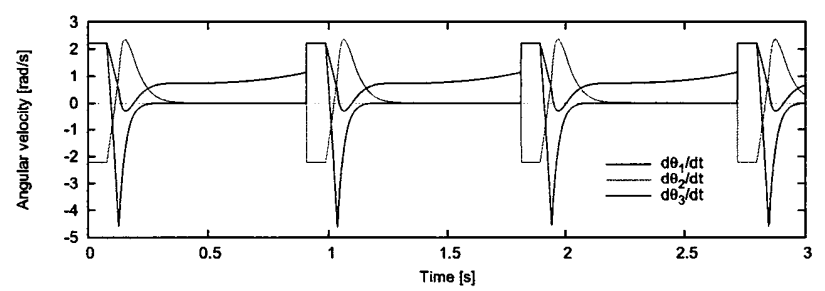

(b) Angular velocity

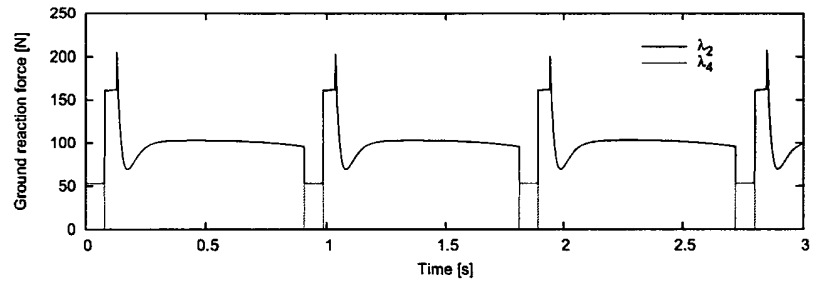

(c) Vertical ground reaction force

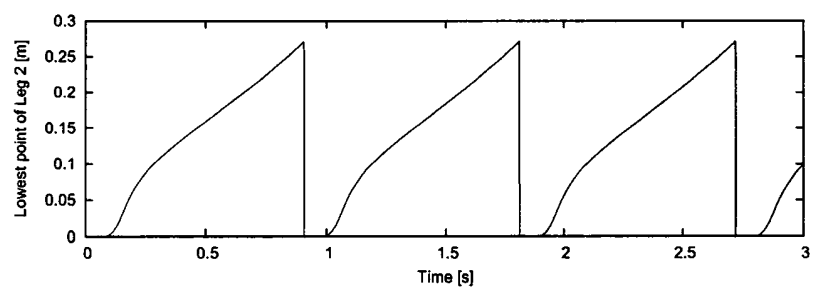

(d) Lowest point of Leg 2

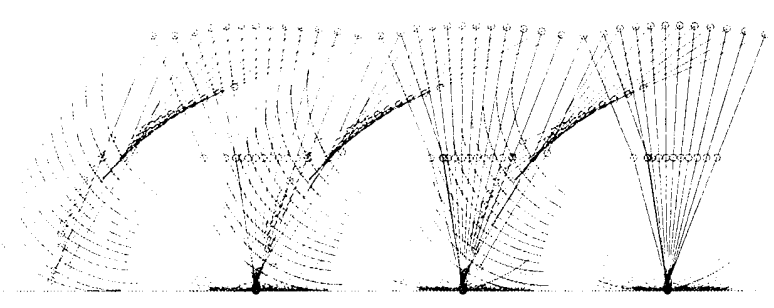

Fig. 3 Stick diagram of steady gait

Table 1 Parameter settings

\begin{tabular}{ccc}
\hline$m_{H}$ & 10.0 & $\mathrm{~kg}$ \\
$m$ & 0.01 & $\mathrm{~kg}$ \\
$\alpha$ & $\pi / 4$ & $\mathrm{rad}$ \\
$R$ & 0.40 & $\mathrm{~m}$ \\
\hline
\end{tabular}

\begin{tabular}{ccc}
\hline$a$ & 0.3 & $\mathrm{~m}$ \\
$b$ & 0.2 & $\mathrm{~m}$ \\
$L_{1}(=a+b)$ & 0.50 & $\mathrm{~m}$ \\
$L_{2}$ & 0.50 & $\mathrm{~m}$ \\
\hline
\end{tabular}

床反力 $\lambda_{2}, \lambda_{4}$ がいずれも正值であること，つまりDLS が維持 されていることが分かる. その後, 制御相 III における PD 制御 が開始され， $\theta_{2} ， \theta_{3}$ がいずれも目標值 $0[\mathrm{rad}]$ へと収束を始めて いる. (a) より, PD 制御が開始されると同時に $\theta_{1}$ が単調増加か らいったん減少を始めることが分かるが，これは Leg 1 の膝関 節が急激に伸長運動を開始したことに起因する現象である. 図 2(d) は Leg 2 の足裏最下点の $Z$ 座標をプロットしたものである が, ゼロから単調に上昇していることが分かる，これは図 3 か らも明らかである.

本稿では省略するが, 膝関節トルクを印可しながら衝突する 場合には $\lambda_{4}^{+}<0$ となるため, 瞬間的でない DLS は発現しない ことを付記しておく.

\section{5. まとめと今後の課題}

本稿では, DLS の発現可能性を持つ歩行モデルとして能動・ 受動の切り替えが可能な膝関節を持つ RW を考え, 衝突時から 膝を自由関節にすることで実際に DLS が発現することを数值シ ミュレーションを通して示した，今後は更に解析を進め, 物理パ ラメータや身体形状とDLS の発現傾向との関係を明らかにして いく必要がある.

McGeer は受動歩行運動において膝折れ現象が発生すること を実験的に示したが $[1][2]$, 動画を良く観察すると, そのタイミ ングは衝突直後であることに気付く．本稿の結果を踏まえてこ れを顧みると, 膝折れ現象は前脚が着地しても後脚が離陸しな いために発生する，と説明することができる，今後はこの仮説の 検証も進めていく予定である.

\section{参考文献}

[1] T. McGeer, "Passive dynamic walking," Int. J. of Robotics Research, Vol. 9, No. 2, pp. 62-82, 1990.

[2] T. McGeer, "Passive walking with knees," Proc. of the IEEE Int. Conf. on Robotics and Automation, Vol. 3, pp. 1640-1645, 1990.

[3] 浅野, 河本, “粘弾性脚を持つリムレスホイールの受動歩行”, 第 29 回日本ロボット学会学術講演会予稿集, 3J1-5, 2011.

Fig. 2 Simulation results of level walking 